\title{
Markierungsfreie Optische Detektion von Schmerzmitteln in Fließgewässern
}

\author{
Sabrina Rau ${ }^{1}$, Norbert Scheibe ${ }^{2}$, Urs Hilbig ${ }^{1}$, Wolf von Tümpling ${ }^{2}$, Günter Gauglitz ${ }^{1}$ \\ ${ }^{1}$ Eberhard Karls Universität, Institut für Physikalische und Theoretische Chemie, Auf der Morgenstelle \\ 15, 72076 Tübingen, Deutschland \\ sabrina.rau@uni-tuebingen.de \\ ${ }^{2}$ Helmholtz-Zentrum für Umweltforschung, Gewässeranalytik und Chemometrie, Brückstraße 3a, \\ 39114 Magdeburg, Deutschland
}

\begin{abstract}
Pharmakologisch aktive Stoffe wie Hormone, Antibiotika, Antirheumatika, Betablocker oder Rötgenkontrastmittel, reichern sich zunehmend in der Umwelt an. In Oberflächengewässer gelangen sie vor allem durch ihre unsachgerechte Entsorgung oder durch menschliche oder tierische Ausscheidungen, da Arzneimittel bei der Abwasserreinigung in Kläranlagen nur unvollständig entfernt werden.

Um Mensch, Tier und Umwelt schützen zu können, ist es unumgänglich geeignete Nachweis- und Quantifizierungsmethoden zu entwickeln, die auch in komplexen Matrices wie Flusswasser sensitiv und selektiv messen können.

In der hier vorgestellten Arbeit wurde mittels der markierungsfreien und zeitaufgelösten Reflektometrischen Interferenzspektroskopie (RIfS) ein direkt optischer Immunosensor exemplarisch für das Schmerzmittel Diclofenac entwickelt, mit dessen Hilfe dieses in Flusswasser nachgewiesen und quantifiziert werden kann. Es konnten gute Wiederfindungsraten sowohl in gespiktem filtrierten, als auch in gespiktem unfiltrierten Flusswasser der „Nagold“ erhalten werden. Zudem wurden Matrixeffekte der komplexen Matrix Flusswasser am Beispiel von Huminsäuren untersucht.
\end{abstract}

Key words: Schmerzmittel, Fließgewässer, optischer Biosensor, markierungsfrei, Immunoassay

\section{Einführung}

Neben seit längerem bekannten Schadstoffen wie Schwermetallen, Industriechemikalien oder Pestiziden, reichern sich auch pharmakologisch aktive Stoffe zunehmend in der Umwelt an $[1,2]$.

Wie Arzneimittel auf Menschen wirken ist gut untersucht. Um eine Gefährdung für die Umwelt abschätzen zu können, können diese Daten jedoch nicht herangezogen werden, denn Arzneimittel beeinflussen z.B. in Fischen, Mückenlarven oder Algen ganz andere Mechanismen, als in Säugetieren oder Menschen. Ökosysteme können demzufolge schon durch Arzneimittelrückstände in geringen Konzentrationen potentiell geschädigt oder zumindest gestört werden. Jedoch liegen bisher kaum Langzeitstudien zum Einfluss dieser Kontaminatoren auf die Umwelt vor. Dennoch wird zunehmend deutlich [3-5], dass ein Monitoring zwingend von Nöten ist.
$\mathrm{Zu}$ diesem Schluss kommt auch eine Literaturstudie, die 2011 vom Umweltbundesamt in Auftrag gegeben wurde. Die Studie ist eine aktuelle Bestandsaufnahme der in Deutschland und dem europäischen Ausland vorliegenden Monitoringdaten zum Vorkommen und Verhalten von Arzneimitteln in der Umwelt. Dabei wurden nicht nur gemessene Umweltkonzentrationen, sondern auch Ergebnisse ökotoxikologischer und physikochemischer Untersuchungen berücksichtigt. Unter den 24 Pharmazeutika, welche in dieser Studie hoch prioritär eingestuft wurden, befindet sich auch das nichtsteroidale Antirheumatikum Diclofenac [6].

Auch die Europäische Union hat auf die Problematik der Anreicherung pharmakologisch aktiver Substanzen in Oberflächengewässern mit einer Änderung der Europäischen Wasserrahmenrichtlinie reagiert. Diclofenac, 17-beta-Östradiol und 17-alpha-Ethinylöstradiol 
wurden als erste Arzneimittelstoffe in die Beobachtungsliste aufgenommen [7].

Diclofenac wird in großen Mengen in der Human- und Veterinärmedizin eingesetzt, da es analgetisch, antiphlogistisch und antipyretisch wirkt [8]. Es wird mittlerweile in Spurenkonzentrationen in vielen Oberflächengewässern gefunden und steht unter Verdacht Nierenschäden in Fischen hervorzurufen [6,9].

Um die Umwelt schützen zu können, ist es unumgänglich geeignete Nachweis- und Quantifizierungsmethoden zu entwickeln. Obwohl optische Biosensoren viele Vorteile gegenüber konventionellen Methoden, wie eine geringe Probenvorbereitung, verkürzte Analysezeit sowie die Möglichkeit von vor-Ort Messungen bieten, sind bisher kaum optische Biosensoren zum Nachweis von Diclofenac in Fließgewässern in der Literatur beschrieben.

In der hier vorgestellten Arbeit wurde mittels der markierungsfreien und zeitaufgelösten Reflektometrischen Interferenzspektroskopie (RIfS) ein direkt optischer Immunosensor entwickelt, mit dessen Hilfe Diclofenac in Flusswasser nachgewiesen und quantifiziert werden kann.

Flusswasser ist eine komplexe Probenmatrix, da in ihr eine Vielzahl an gelösten und ungelösten Stoffen vorhanden ist, die alle potentiell Matrixeffekte verursachen können. Markierungsfreie optische Detektionsmethoden werden deutlich stärker von Matrixeffekten beeinflusst wie solche, die mit Markierungen arbeiten. Dennoch ist es von Vorteil markierungsfreie optische Detektionsmethoden zu verwenden, da diese durch Vermeidung von Markierungsschritten schneller und kostengünstiger sind.

Bisher publizierte Immunoassays, welche sich mit der Detektion und Quantifizierung von Schadstoffen in Fließgewässern beschäftigen, weisen alle auf das hohe Interferenzpotential von Huminsäuren hin [10,11]. Aus diesem Grund wurde der Effekt verschiedener Huminsäure-Konzentrationen auf den hier vorgestellten Immunoassay untersucht.

\section{Methoden}

Als Assayformat wurde der Bindungshemmtest verwendet. Dabei wird die Probe, welche den nachzuweisenden Analyt (Diclofenac) enthält, mit einer definierten Menge des Antikörpers (anti-Diclofenac) für 30 Minuten inkubiert und anschließend über die Oberfläche geleitet. Der Antikörper kann nur noch mit den verbliebenen freien Bindungsstellen an das auf der Oberfläche immobilisierte Antigen bzw. Antigen-Derivat binden (Abb. 1).

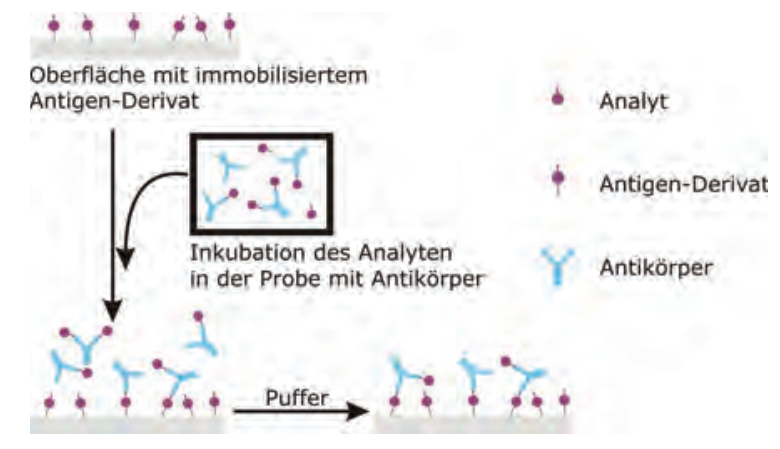

Abb. 1. Schematische Darstellung des Bindungshemmtests.

Für eine hohe Analytkonzentration in der Probe wird demzufolge ein niedriges, für eine niedrige Analytkonzentration ein hohes Messsignal erhalten (Abb. 2).

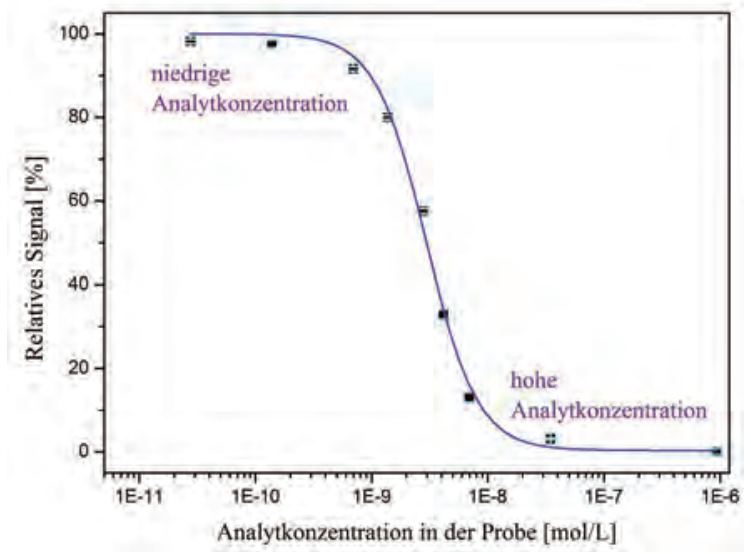

Abb. 2. Darstellung des Zusammenhangs zwischen Analytkonzentration in der Probe und dem detektierten Signal am Beispiel einer Kalibrierkurve.

Da die Immunoreaktion an heterogener Phase stattfindet, kommt den Eigenschaften der Sensoroberfläche eine große Bedeutung zu. Die Sensoroberfläche soll eine hohe Zahl an Bindungsstellen bereit stellen und sie soll möglichst stabil sein, sowohl im Hinblick auf die Probenmatrix, als auch auf die Temperatur oder den $\mathrm{pH}$ Wert. Zudem soll sie mehrfach verwendbar und somit regenerierbar sein. Das allerwichtigste Kriterium stellt jedoch die Vermeidung unspezifischer Wechselwirkungen mit der Probe und somit die Reduzierung von Matrixeffekten dar [12]. Die oben beschrie- 
benen Anforderungen konnten mit der kovalenten Immobilisierung einer Polymerschicht (Polyethylenglycol, PEG) und der anschließenden kovalenten Immobilisierung von Aceclofenac (Diclofenac-Derivat) auf dem Transducer erreicht werden.

Als Detektionsmethode wurde die markierungsfreie, zeitaufgelöste Reflektometrische Interferenzspektroskopie verwendet. Diese beruht auf dem Prinzip der Mehrfachreflexion von Weißlicht an dünnen Schichten [13].

\section{Ergebnisse}

Flusswasser stellt eine komplexe Probenmatrix dar, deren Bestandteile potentiell mit dem Immunoassay interferieren können. Aus diesem Grund wurde der Immunosensor zuerst in Phosphat-Puffer etabliert. Die Parameter des Immunoassays, wie zum Beispiel die Sensoroberfläche, wurden im Bezug auf Spezifität, Reproduzierbarkeit (Abb. 3) und Regenerierbarkeit optimiert.

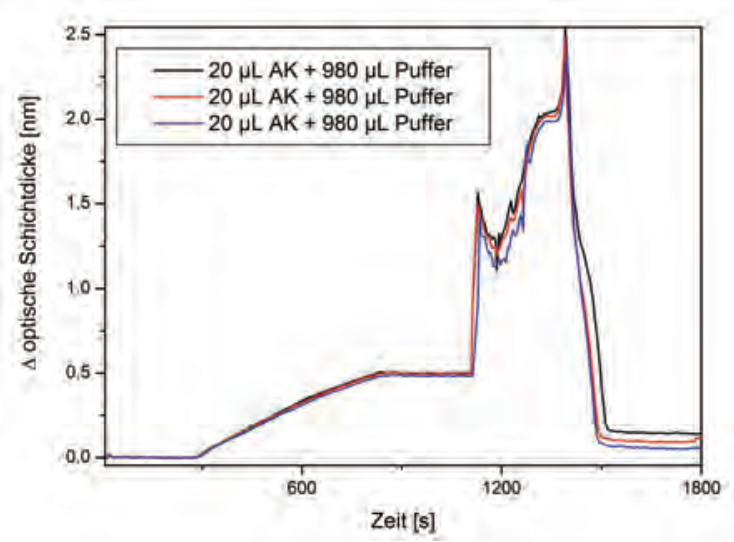

Abb. 3. Dreifachmessung von $20 \mu \mathrm{L}$ anti-Diclofenac. Die Oberfläche wurde mit 0,5 \%iger Natriumdodecylsulfat-Lösung $(\mathrm{pH} 2,2)$ regeneriert.

Der Sensor wurde in Puffer kalibriert und Wiederfindungsraten für vier DiclofenacKonzentrationen bestimmt. Diese liegen zwischen $90-110 \%$ und somit im geforderten Bereich $(70-120 \%)$.

Nach erfolgreicher Optimierung, konnte der Immunoassay in ultrafiltriertem entsalztem Wasser kalibriert werden. Da Antikörper empfindliche biologische Moleküle darstellen, die in Wasser als Probenmatrix möglicherweise nach einiger Zeit denaturieren, musste die Messroutine geringfügig modifiziert werden. Zur Validierung der Kalibrierkurve wurden Wiederfindungsraten für vier Diclofenac-
Konzentrationen bestimmt, die im geforderten Bereich zwischen $70-120 \%$ lagen.

Des Weiteren wurde der Einfluss von verschiedenen Huminsäure-Konzentrationen auf die Oberfläche, den Antikörper sowie den Analyt und somit auf die Analyt-Antikörper Wechselwirkung untersucht. Bis zu einer Huminsäure-Konzentration von $1,5 \mathrm{mg} / \mathrm{L}$ konnte kein Einfluss beobachtet werden.

Filtriertes Flusswasser der Nagold wurde mit verschiedenen Diclofenac-Konzentrationen gespikt und vermessen (Abb. 4). In nichtgespikten Proben war die DiclofenacKonzentration unter der Nachweisgrenze der hier beschriebenen Methode.

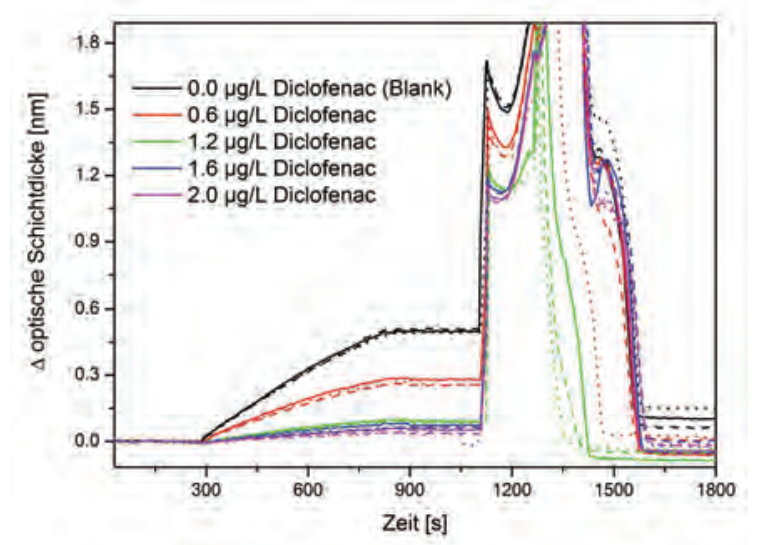

Abb. 4. Bindungskurven; $20 \mu L$ anti-Diclofenac inhibiert mit 0,0 $\mu \mathrm{g} / \mathrm{L} ; 0,6 \mu \mathrm{g} / \mathrm{L} ; 1,2 \mu \mathrm{g} / \mathrm{L} ; 1,6 \mu \mathrm{g} / \mathrm{L} ; 2,0$ $\mu \mathrm{g} / \mathrm{L}$ Diclofenac (Dreifachmessungen).

Für alle vermessenen Konzentrationen konnten gute Wiederfindungsraten erhalten werden (Abb. 5).

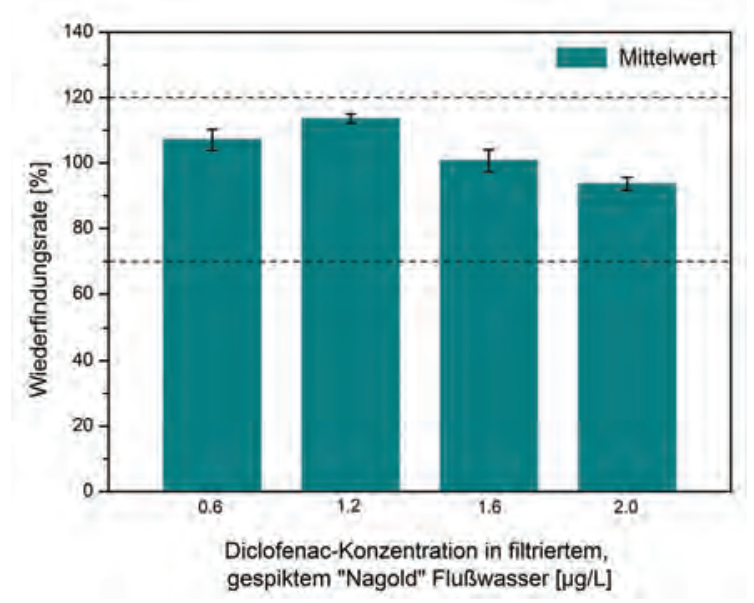

Abb. 5. Wiederfindungsraten in filtriertem, gespiktem "Nagold" Flusswasser. Es wurden vier DiclofenacKonzentrationen vermessen: $0,6 \mu \mathrm{g} / \mathrm{L} ; 1,2 \mu \mathrm{g} / \mathrm{L} ; 1,6$ $\mu \mathrm{g} / \mathrm{L} ; 2,0 \mu \mathrm{g} / \mathrm{L}$ Diclofenac (Dreifachmessungen). 
Um Probenvorbereitungsschritte zu vermeiden, wurde untersucht, ob Messungen auch in unfiltriertem, gespiktem "Nagold“ Flusswasser möglich sind. Dazu wurden Wiederfindungsraten für verschiedene DiclofenacKonzentrationen bestimmt. Diese liegen zwischen $100-115 \%$ und somit im geforderten Bereich (70 -120\%).

Bei dem entwickelten Immunosensor sind demzufolge keine Probenvorbereitungsschritte von Nöten.

\section{Ausblick}

Ein markierungsfreier optischer Biosensor für den Nachweis und die Quantifizierung von Diclofenac in Fließgewässern konnte erfolgreich entwickelt und optimiert werden. Es konnte gezeigt werden, dass die komplexe Matrix für den Immunoassay kaum eine Rolle spielt und eine Probenvorbereitung des Flusswassers nicht von Nöten ist.

In weiteren Schritten soll mittels dieses Immunosensors Diclofenac in Abwasser von Abwasserreinigungsanlagen nachgewiesen und quantifiziert werden.

Zudem sollen die gewonnenen Erkenntnisse auf die im Weiteren zu entwickelnden Immunoassays zum Nachweis und zur Quantifizierung weiterer Schmerzmittel (z.B. Ketoprofen) in Fließgewässern übertragen werden.

\section{Literatur}

[1] D.W. Kolpin et al., Pharmaceuticals, hormones, and other organic wastewater contaminants in U.S. streams, 1999-2000: a national reconnaissance, Environ Sci Technol 36, 12021211 (2002); doi: 10.1021/es011055j

[2] M. Petrovic et al., Endocrine disrupting compounds and other emerging contaminants in the environment: A survey on new monitoring strategies and occurrence data, Anal Bioanal Chem 378, 549-562 (2004); doi: 10.1007/s00216003-2184-7

[3] M. Saravanan, M. Ramesh, Short and long-term effects of clofibric acid and diclofenac on certain biochemical and ionoregulatory responses in an Indian major carp, Cirrhinus mrigala, Chemosphere 93, 388-396 (2013); doi: 10.1016/j.chemosphere.2013.05.015

[4] G.E. Swan et al., Toxicity of diclofenac to Gyps vultures, Biol Lett 2, 279-282 (2006); doi: 10.1098/rsbl.2005.0425
[5] Q. Teng et al., Impacts of $17 \alpha$-ethynyestradiol exposure on metabolite profiles of zebrafish (Danio rerio) liver cells, Aquat Toxicol 130-131, 184-191 (2013); doi: 10.1016/j.aquatox.2013.01.011

[6] A. Bergmann et al., Zusammenstellung von Monitordaten zu Umweltkonzentrationen von Arzneimitteln, Umweltbundesamt (2011)

[7] Richtlinie 2013/39/EU des Europäischen Parlaments und des Rates vom 12. August 2013 zur Änderung der Richtlinien 2000/60/EG und 2008/105/EG in Bezug auf prioritäre Stoffe im Bereich der Wasserpolitik (2013)

[8] E.C. Ku, J.M. Wasvary, W.D. Cash, Diclofenac sodium (GP 45840, Voltaren), a potent inhibitor of prostaglandin synthetase, Biochem Pharmacol 24, 641-643 (1975)

[9] J. Schwaiger et al., Toxic effects of the nonsteroidal anti-inflammatory drug diclofenac Part I: histopathological alterations and bioaccumulation in rainbow trout, Aquat Toxicol 68, 141-150 (2004); doi: 10.1016/j.aquatox.2004.03.014

[10] A. Deng et al., Residue analysis of the pharmaceutical diclofenac in different water types using ELISA and GC-MS, Environ Sci Technol 37, 3422-3429 (2003); doi: 10.1021/es0341945

[11] E. Brun et al., Evaluation of a novel malathion immunoassay for groundwater and surface water analysis, Environ Sci Technol 39, 2786-2794 (2005); doi: 10.1021/es048945u

[12] S. Rau, G. Gauglitz, Reflektometric interference spectroscopy (RIfS) as a new tool to measure in the complex matrix milk at low analyte concentration, Anal Bioanal Chem 402, 529-536 (2012); doi: 10.1007/s00216-011-5470-9

[13] G. Gauglitz, Direct optical detection in bioanalysis: an update, Anal Bioanal Chem 398, 2363-2372 (2010); doi: 10.1007/s00216-0103904-4 